\title{
Assessment of Malnutrition in Community Chinese Elderly: A Hidden Problem in a Developed Society
}

\author{
Josette Sin-yee Chor, ${ }^{1}$ Jason Leung, ${ }^{2}$ Sian Griffiths, ${ }^{1}$ and Ping-chung Leung ${ }^{2}$ \\ ${ }^{1}$ Jockey Club School of Public Health and Primary Care, The Chinese University of Hong Kong, Room 202, \\ School of Public Health Building, Prince of Wales Hospital, Shatin, Hong Kong \\ ${ }^{2}$ Jockey Club Centre for Osteoporosis Care and Control, The Chinese University of Hong Kong, Room 318, \\ School of Public Health Building, Prince of Wales Hospital, Shatin, Hong Kong \\ Correspondence should be addressed to Josette Sin-yee Chor; josette@cuhk.edu.hk
}

Received 8 August 2012; Accepted 29 August 2012

Academic Editors: D. Chan and O. Weinreb

Copyright (C) 2013 Josette Sin-yee Chor et al. This is an open access article distributed under the Creative Commons Attribution License, which permits unrestricted use, distribution, and reproduction in any medium, provided the original work is properly cited.

\begin{abstract}
Background. Malnutrition can lead to chronic disease especially in vulnerable population. This study is to explore the extent of the malnutrition problem in community Chinese elderly and its associating factors in Hong Kong. Method. This is a cross-sectional study using structured interview with 7-day food frequency questionnaires. 1960 men and 1954 women aged 65 or above were recruited from the community. Results. Less than half of the participants fulfilled the recommended intake level of the others. Less than $10 \%$ of participants met the daily requirement of fibre, Calcium, Vitamin D, Iodine, and Copper. $22.32 \%$ of male participants and $6.21 \%$ of female participants have cholesterol intake exceeding the recommended upper level. $41.19 \%$ men and $28.99 \%$ women exceeded the upper level of fat percentage of total energy. Educational level was demonstrated to have a consistent positive association with the adequate intake in most nutrients. Single or divorced marital status is a risk factor in over-intake of saturated fatty acid in men and under-intake in Zinc and meat in women. Conclusion. Our study has shown the serious malnutrition problem in a wide range of nutrients in community Chinese elderly in Hong Kong.
\end{abstract}

\section{Introduction}

Nutrition is vital for healthy ageing. Inappropriate diet is associated with different illnesses such as cancer, stroke, ischaemic heart disease, diabetic mellitus, and osteoporosis irrespective of the sex and ethnicity [1]. Previous studies have shown that the Mediterranean diet helped to reduce risk of cancer, which suggested that increasing fruit and vegetable consumption and decreasing meat intake would have a protective effect against cancer [2]. Another study also showed a strong protective effect of fibre, Vitamin C, and carotene as well as positive association between dietary fat intake and breast cancer amongst the Chinese [3]. A systematic review showed that diets with nonhydrogenated unsaturated fat, as the major forms of fat intake, whole grain, as the major form of carbohydrate along with high intake of fruits and vegetables, as well as adequate omega- 3 fatty acid had a significant protection effect against cardiovascular diseases [4]. Another study in China showed that hyperlipidemia was positively associated with total energy, total fat, and saturated fatty acid intake and negatively associated with fibre intake [5]. A previous local study in Hong Kong found reduced mortality with increased fish uptake [6]. Inappropriate nutrient intake is not only confined to developing countries, but is also common amongst elderly in high income countries [7]. It is a global health issue closely related to socioeconomic transition and social disparity $[7,8]$. Both over- and undernutrition can exist at the same time in the same country among different socioeconomic classes $[7,8]$. This leads to differentials in mortality and morbidity in a population especially in the vulnerable groups such as elderly.

Although the importance of an appropriate diet is well documented, the prevalence of poor nutrition including a detailed account of specific nutrient intake is not well described in Chinese elderly populations. Appropriate health education and policies cannot be formulated without this 
important information. Such studies as have been carried out have found variable results. For example, a small survey done in Singapore showed that elderly do not conform to healthy eating guidelines for the major food categories [9]. The only study assessing the protein intake of elderly people in a lower socioeconomic group in Hong Kong was done 20 years ago [10].

Thus, this study was to assess the health need in elderly people by documenting the extent of the malnutrition problem in a community of Chinese elderly and study the associated factors in Hong Kong.

\section{Materials and Methods}

2.1. Study Design and Participants. Two thousand men and two thousand women aged 65 or above were invited from the community by self-referral in health talks and posting recruitment notice in community centers for the elderly and housing estates in Hong Kong. All participants are Chinese and community dwelling. They were recruited and followed up for 5 years with questionnaires and health checks including blood taking, blood pressure monitoring, cognitive function assessment, and bone density measurement.

2.2. Measurements. Sociodemographics including age, educational level, marital status, and medical history were recorded by structured questionnaires administered by trained staff. Socioeconomic status was measured by a selfrated 10-point ladder scale. Their body mass index (BMI) was measured.

Dietary intake was assessed by a 7-day food frequency questionnaire with mean nutrients per day calculated using the table derived from McCance and Widdowson and the Chinese Medical Science Institute $[11,12]$. The food frequency questionnaire consisted of seven categories of food items including bread/pasta/rice, vegetables, fruits, meat, beverages, dimsum/snacks, soups, and oil/salt/sauce. Food items in each category were chosen based on the Chinese diet in the Hong Kong population. Methods of cooking were also recorded. Since a previous study had found that variation between seasons was not a significant factor, we conducted interviews throughout the year regardless of the season [13]. Nutrients and food items of interest are Vitamin A, Vitamin B1, B2, and B3, Vitamin C, Vitamin D, Calcium, Phosphorus, Iron, Zinc, Iodine, Copper, protein, carbohydrate, fruit, vegetable, meat, fibre, cholesterol, fat percentage of total energy, saturated fatty acid, monosaturated fatty acid and polyunsaturated fatty acid, and total energy.

The subjects were asked to fill in the food items consumed, portion size, and number of times consumed per day, per week, or per year for less frequently consumed items. Portion size was explained to the participants by pictures and photographs. The amount of oil used in cooking is estimated by the formula used in another local study depending on the method of cooking: 0.2 tablespoon (tbsp) for steaming fish or stir frying half a portion of vegetables; 1 tbsp for stir frying one portion of meat or one portion of vegetables [14]. Validity of this food frequency questionnaire was validated by comparing the 24-hour intake of total energy, lipid, sodium, and potassium, with estimated values of total energy expenditure, plasma lipid level, and 24-hour urinary sodium, and potassium outputs. There were no significant differences observed in energy intake but there was an underestimation of sodium and potassium. The lipid intake is highly associated with the plasma lipid level [14].

2.3. Statistical Analysis. The mean dietary intake of each nutrient was calculated and compared with the intake level recommended by Department of Health, Hong Kong, and we have used the United States recommended levels when local standards were not available. The percentage of participants under or exceeding the recommended level was calculated. The adjusted odds ratio by multiple logistic regression was used to identify the nutritional risk factor of various sociodemographics and BMI of our subjects in different nutrients.

\section{Results}

One thousand nine hundred and sixty men and one thousand nine hundred and fifty four women aged 65 or above were recruited from the community. Their mean BMI was not significantly different from the Hong Kong population average in this age group $\left(23 \mathrm{~kg} / \mathrm{m}^{2}\right)$. The characteristics were shown in Table 1 . The mean nutrient intakes of the subjects were shown in Tables 2 and 3. Most participants met the recommended requirement of Vitamin A; Vitamin C, Phosphorus, Iron, protein, and carbohydrate. There was no significant difference in men and women. A small percentage of participants exceeded the upper recommended level, most commonly for Vitamin A for which 3.3\% of men and $2.3 \%$ of women exceeded the upper intake level of $3000 \mu \mathrm{g}$.

Less than half of the participants fulfilled the recommended intake level of the other nutrients. The most serious ones were Vitamin D, Iodine, and Copper for which none of the participants met the recommended daily intake. Moreover, less than $5 \%$ of subjects have taken enough Calcium and less than $10 \%$ of participants met the daily requirement of fibre. Less than half of the participants met the recommended daily intake of the following nutrients: Vitamin B1, Vitamin B2, Vitamin B3, Zinc, fruit, vegetable, and meat. These findings show that in most cases, more elderly women are undernourished than men.

Vitamin B3 intake showed the greatest variation, and despite less than half of the participants reaching the recommended level, up to $10 \%$ of subjects exceeded the upper recommended level (12.66\% in men and $6.01 \%$ in women).

Fat intake was also suboptimal. Hong Kong elderly overconsumed fat. The situation in men is worse than in women. $22.32 \%$ of male participants and $6.21 \%$ of female participants had cholesterol intakes exceeding the recommended upper level. $41.19 \%$ men and $28.99 \%$ women exceeded the upper level of fat as a percentage of total energy. The picture is better for saturated fatty acid as a percentage of total energy. Only $5.11 \%$ of men and $2.4 \%$ of women exceeded the upper recommended level.

Logistic regression analysis of socioeconomic factors such as age, socioeconomic status, educational level, marital 
TABLE 1: Characteristics of subjects.

\begin{tabular}{|c|c|c|c|}
\hline \multirow{2}{*}{ Variable } & \multicolumn{2}{|c|}{ Mean (SD)/Frequency (\%) } & \multirow{2}{*}{$P$ value ${ }^{*}$} \\
\hline & Female & Male & \\
\hline Age & $72.58(5.36)$ & $72.39(5.01)$ & 0.2541 \\
\hline Social economic ladder of Hong Kong & $4.64(1.92)$ & $4.45(1.87)$ & 0.0020 \\
\hline Education level & & & $<0.0001$ \\
\hline No education & $753(37.65 \%)$ & $103(5.15 \%)$ & \\
\hline Primary or below & $903(45.15 \%)$ & $1104(55.20 \%)$ & \\
\hline Secondary or above & $344(17.20 \%)$ & $793(39.65 \%)$ & \\
\hline Single, divorced, or widowed & $931(46.55 \%)$ & $240(12.00 \%)$ & $<0.0001$ \\
\hline Living alone & $341(17.05 \%)$ & $92(4.60 \%)$ & $<0.0001$ \\
\hline Housewife & $206(10.30 \%)$ & - & \\
\hline Weight & $54.52(8.50)$ & $62.44(9.38)$ & $<0.0001$ \\
\hline Height & $150.92(5.32)$ & $163.08(5.72)$ & $<0.0001$ \\
\hline BMI & & & 0.1081 \\
\hline Underweight $(<18.5)$ & $100(5.00 \%)$ & $115(5.75 \%)$ & \\
\hline Normal $(18.5-<23)$ & $711(35.55 \%)$ & $760(38.00 \%)$ & \\
\hline Overweight $(\geq 23)$ & $1189(59.45 \%)$ & $1125(56.25 \%)$ & \\
\hline
\end{tabular}

${ }^{*} P$ value of $t$-test for continuous and $\chi^{2}$ for categorical variables.

status, and BMI found that only educational level had a consistently positive association with adequate intake of most nutrients (Table 4). Single or divorced marital status is a risk factor in over-intake of saturated fatty acid of total energy in men. It is also a risk factor of under-intake in zinc and meat in women (Table 5). There is no association between BMI, age, living and socioeconomic status, and nutritional status. Therefore, body weight cannot reflect whether the subject is having the appropriate diet or not. The undernutrition was more serious when age advanced in both men and women (Tables 6 and 7).

\section{Discussion}

Our results showed that malnutrition exists amongst the elderly in Hong Kong Chinese elderly. They have a low intake of fibre, Calcium, and Vitamin D. On the other hand, they have an overconsumption of fat and cholesterol. Their diets are not appropriate for their needs, possibly contributing to the burden of chronic disease and/or poor control of existing illnesses such as osteoporosis, cardiovascular disease, and high fat diet-related cancer. This is reflected by the ongoing upward trend in age standardized cancer rate in breast cancer and male colorectal cancer which are most closely contributed by inappropriate dietary intake [15]. The International Osteoporosis Foundation also pointed out that the hip fracture rate in Hong Kong has increased by $300 \%$ from the 1960s to the 1990s and now costs more than 17 million USD a year in acute hospital care [16]. The coronary heart disease has also risen from $38.6 \%$ in 1972 to $59.6 \%$ in 1992 [17]. It is also now the second commonest cause of death in Hong Kong. Comparison with another local study done 20 years ago showed that the protein intake has greatly improved in both men and women reflecting the increasing affluence of our society [10].

Our study showed that people with lower education level, single/divorced/widowed, or male gender are at higher nutritional risk. These findings were compatible with another study of the Chinese population in Singapore [9]. However, there is no increased risk with advanced age and or with lower socioeconomic level which is at variance with other studies, possibly reflecting the greater socioeconomic homogeneity in Hong Kong. Another study that showed BMI has a positive association with the nutritional status. However, in our study, there is no association. This is perhaps because the energy from fat is quite high in our subjects and therefore the BMI cannot be used as a proxy measurement of overall nutrition.

Factors contributing to the nutritional imbalance are complex. A previous study has demonstrated that Chinese elderly have poor nutritional knowledge and they held strong traditional Chinese or food-texture-related dietary restriction attitudes [18]. This study also showed elderly of younger age and higher educational level had better nutrition knowledge. Therefore, health and nutrition liturgy is very important in strategies for promoting health in the elderly. We did not ask the source of food. However, in Chinese culture, elderly people usually cook and eat at home. Therefore, a health promotion programme targeted at healthy home or healthy recipe can help solving this problem in elderly.

Our study has its own limitations. The use of food frequency questionnaires has the merit of alleviating the memory burden amongst the elderly and can be used to assess long-term diet and is less influenced by daily variation. The tool has also been validated and used in nutrition studies in Chinese populations. However, it cannot cover all the 
TABLE 2: Median (IQR) nutrient intake of the male participants.

\begin{tabular}{|c|c|c|c|c|c|}
\hline Nutrient per day & Median (IQR) & $\begin{array}{l}\text { Recommended } \\
\text { intakes }\end{array}$ & $\begin{array}{l}\text { Percentage meeting } \\
\text { the recommendation }\end{array}$ & $\begin{array}{c}\text { Tolerable upper intake } \\
\text { levels }\end{array}$ & $\begin{array}{c}\text { Percentage } \\
\text { over the } \\
\text { upper intake }\end{array}$ \\
\hline Vitamin A ( $\mu \mathrm{g})$ & $929.47(649.92,1,322.95)$ & $900^{\mathrm{a}}$ & $52.25 \%$ & $3000^{\mathrm{a}}$ & $3.30 \%$ \\
\hline Vitamin B1 (mg) & $0.87(0.67,1.17)$ & $1.2^{\mathrm{b}}$ & $22.77 \%$ & & \\
\hline Vitamin B2 (mg) & $0.94(0.70,1.27)$ & $1.3^{\mathrm{b}}$ & $23.62 \%$ & & \\
\hline $\begin{array}{l}\text { Vitamin B3/niacin } \\
(\mathrm{mg})\end{array}$ & $15.05(11.18,22.89)$ & $16^{\mathrm{b}}$ & $45.10 \%$ & $35^{\mathrm{b}}$ & $12.66 \%$ \\
\hline Vitamin C (mg) & $140.10(98.73,195.44)$ & $90^{\mathrm{c}}$ & $79.58 \%$ & $2000^{c}$ & $0 \%$ \\
\hline Vitamin D $(\mu \mathrm{g})$ & $0.19(0.07,0.41)$ & $10^{\mathrm{d}}$ & $0 \%$ & $50^{\mathrm{d}}$ & $0 \%$ \\
\hline Calcium (mg) & $575.80(411.98,779.35)$ & $1200^{\mathrm{d}}$ & $4.40 \%$ & $2500^{\mathrm{d}}$ & $0 \%$ \\
\hline Phosphorous (mg) & $\begin{array}{c}1,058.23(806.95 \\
1,424.99)\end{array}$ & $700^{\mathrm{d}}$ & $84.38 \%$ & $3000^{\mathrm{d}}$ & $0.30 \%$ \\
\hline Iron (mg) & $14.56(11.12,19.19)$ & $8^{\mathrm{a}}$ & $92.94 \%$ & $45^{\mathrm{a}}$ & $0.35 \%$ \\
\hline Zinc (mg) & $9.62(7.47,11.96)$ & $11^{\mathrm{a}}$ & $33.73 \%$ & $40^{\mathrm{a}}$ & $0 \%$ \\
\hline Iodine $(\mu \mathrm{g})$ & $0.29(0.10,1.06)$ & $150^{\mathrm{a}}$ & $0.10 \%$ & $1100^{\mathrm{a}}$ & $0 \%$ \\
\hline Copper $(\mu \mathrm{g})$ & $0.12(0.06,0.23)$ & $900^{\mathrm{a}}$ & $0 \%$ & $10000^{\mathrm{a}}$ & $0 \%$ \\
\hline Total energy (Kcal) & $\begin{array}{c}2,037.84(1,675.09 \\
2,457.42)\end{array}$ & $2330 / 2100^{\mathrm{e}}$ & $36.39 \%$ & & \\
\hline Protein (g) & $80.97(62.24,106.03)$ & $56^{\mathrm{f}}$ & $82.48 \%$ & & \\
\hline $\begin{array}{l}\text { Protein }(\mathrm{g}) / \text { body } \\
\text { weight }(\mathrm{kg})\end{array}$ & $1.32(1.00,1.74)$ & $0.8^{\mathrm{f}}$ & $88.59 \%$ & & \\
\hline $\begin{array}{l}\text { Protein \% of total } \\
\text { energy }\end{array}$ & $16.14(13.91,18.74)$ & & & $35^{\mathrm{f}}$ & $0 \%$ \\
\hline Carbohydrate (g) & $274.43(225.50,342.72)$ & $130^{\mathrm{f}}$ & $98.55 \%$ & & \\
\hline Fruit (g) & $231.62(154.67,344.45)$ & $300^{g}$ & $34.08 \%$ & & \\
\hline Vegetable $(\mathrm{g})$ & $208.36(143.70,305.36)$ & $240^{g}$ & $40.84 \%$ & & \\
\hline Meat (g) & $145.97(99.00,215.27)$ & $160^{g}$ & $49.85 \%$ & & \\
\hline Fibre (g) & $8.61(6.21,12.00)$ & $16^{\mathrm{g}}$ & $9.91 \%$ & & \\
\hline Cholesterol (mg) & $200.54(135.01,288.74)$ & & & $300^{\mathrm{h}}$ & $22.32 \%$ \\
\hline Fat $\%$ of total energy & $28.63(24.62,32.95)$ & & & $30^{\mathrm{h}}$ & $41.19 \%$ \\
\hline $\begin{array}{l}\text { Saturated fatty acid \% } \\
\text { of total energy }\end{array}$ & $6.71(5.51,8.02)$ & & & $10^{\mathrm{h}}$ & $5.11 \%$ \\
\hline $\begin{array}{l}\text { Monounsaturated } \\
\text { fatty acid \% of total } \\
\text { energy }\end{array}$ & $10.56(8.87,12.47)$ & & & $12^{\mathrm{h}}$ & $29.78 \%$ \\
\hline $\begin{array}{l}\text { Polyunsaturated fatty } \\
\text { acid \% of total energy }\end{array}$ & $6.78(5.60,8.38)$ & & & $6^{\mathrm{h}}$ & $66.07 \%$ \\
\hline \multicolumn{6}{|c|}{ 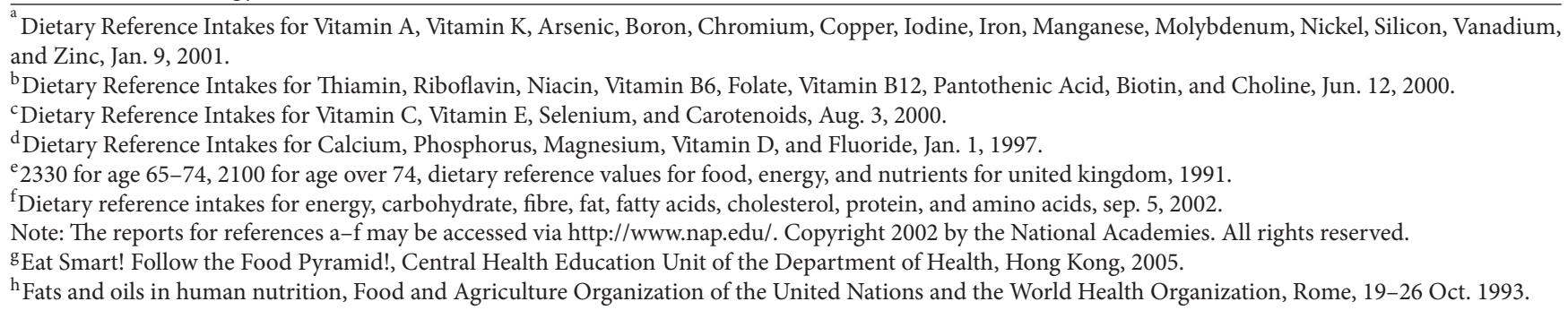 } \\
\hline
\end{tabular}


TABLE 3: Median (IQR) nutrient intakes in female participants.

\begin{tabular}{|c|c|c|c|c|c|}
\hline Nutrient per day & Median (IQR) & $\begin{array}{l}\text { Recommended } \\
\text { intakes }\end{array}$ & $\begin{array}{l}\text { Percentage } \\
\text { meeting the } \\
\text { recommendation }\end{array}$ & $\begin{array}{l}\text { Tolerable upper } \\
\text { intake levels }\end{array}$ & $\begin{array}{l}\text { Percentage over the } \\
\text { upper intake }\end{array}$ \\
\hline Vitamin A $(\mu \mathrm{g})$ & $923.56(669.18,1,266.28)$ & $700^{\mathrm{a}}$ & $71.66 \%$ & $3000^{\mathrm{a}}$ & $2.30 \%$ \\
\hline Vitamin B1 (mg) & $0.77(0.58,1.01)$ & $1.1^{\mathrm{b}}$ & $17.78 \%$ & & \\
\hline Vitamin B2 (mg) & $0.82(0.60,1.11)$ & $1.1^{\mathrm{b}}$ & $26.14 \%$ & & \\
\hline $\begin{array}{l}\text { Vitamin B3/niacin } \\
(\mathrm{mg})\end{array}$ & $10.48(8.08,14.73)$ & $14^{\mathrm{b}}$ & $27.74 \%$ & $35^{\mathrm{b}}$ & $6.01 \%$ \\
\hline Vitamin C (mg) & $140.84(101.17,187.45)$ & $75^{\mathrm{c}}$ & $88.03 \%$ & $2000^{c}$ & $0 \%$ \\
\hline Vitamin D $(\mu \mathrm{g})$ & $0.19(0.08,0.38)$ & $10^{\mathrm{d}}$ & $0 \%$ & $50^{\mathrm{d}}$ & $0 \%$ \\
\hline Calcium (mg) & $527.55(376.39,713.50)$ & $1200^{\mathrm{d}}$ & $2.70 \%$ & $2500^{\mathrm{d}}$ & $0 \%$ \\
\hline Phosphorous (mg) & $886.05(683.21,1,167.16)$ & $700^{\mathrm{d}}$ & $73.01 \%$ & $3000^{\mathrm{d}}$ & $0.10 \%$ \\
\hline Iron (mg) & $11.66(9.19,15.30)$ & $8^{\mathrm{a}}$ & $84.58 \%$ & $45^{\mathrm{a}}$ & $0.30 \%$ \\
\hline Zinc (mg) & $7.38(5.91,9.27)$ & $8^{\mathrm{a}}$ & $40.31 \%$ & $40^{\mathrm{a}}$ & $0 \%$ \\
\hline Iodine $(\mu \mathrm{g})$ & $0.36(0.10,5.53)$ & $150^{\mathrm{a}}$ & $0 \%$ & $1100^{\mathrm{a}}$ & $0 \%$ \\
\hline Copper $(\mu \mathrm{g})$ & $0.07(0.04,0.14)$ & $900^{\mathrm{a}}$ & $0 \%$ & $10000^{\mathrm{a}}$ & $0 \%$ \\
\hline Total energy (Kcal) & $\begin{array}{c}1,504.03(1,262.64, \\
1,843.71)\end{array}$ & $1900 / 1810^{\mathrm{e}}$ & $23.08 \%$ & & \\
\hline Protein (g) & $60.50(46.44,77.45)$ & $46^{\mathrm{f}}$ & $75.81 \%$ & & \\
\hline $\begin{array}{l}\text { Protein }(\mathrm{g}) / \text { body } \\
\text { weight }(\mathrm{kg})\end{array}$ & $1.12(0.85,1.47)$ & $0.8^{\mathrm{f}}$ & $79.07 \%$ & & \\
\hline $\begin{array}{l}\text { Protein } \% \text { of total } \\
\text { energy }\end{array}$ & $15.97(13.72,18.41)$ & & & $35^{\mathrm{f}}$ & $0.15 \%$ \\
\hline Carbohydrate (g) & $216.63(180.31,261.49)$ & $130^{\mathrm{f}}$ & $95.29 \%$ & & \\
\hline Fruit (g) & $212.89(148.19,315.74)$ & $300^{g}$ & $28.14 \%$ & & \\
\hline Vegetable (g) & $207.42(147.10,288.72)$ & $240^{\mathrm{g}}$ & $37.56 \%$ & & \\
\hline Meat (g) & $104.36(69.53,152.35)$ & $160^{\mathrm{g}}$ & $27.69 \%$ & & \\
\hline Fibre (g) & $8.01(5.89,10.58)$ & $16^{\mathrm{g}}$ & $6.11 \%$ & & \\
\hline Cholesterol (mg) & $133.71(89.15,190.70)$ & & & $300^{\mathrm{h}}$ & $6.21 \%$ \\
\hline Fat $\%$ of total energy & $26.75(23.50,30.74)$ & & & $30^{\mathrm{h}}$ & $28.99 \%$ \\
\hline $\begin{array}{l}\text { Saturated fatty acid \% } \\
\text { of total energy }\end{array}$ & $5.92(4.96,7.02)$ & & & $10^{\mathrm{h}}$ & $2.40 \%$ \\
\hline $\begin{array}{l}\text { Monounsaturated } \\
\text { fatty acid \% of total } \\
\text { energy }\end{array}$ & $9.92(8.40,11.89)$ & & & $12^{\mathrm{h}}$ & $23.69 \%$ \\
\hline $\begin{array}{l}\text { Polyunsaturated fatty } \\
\text { acid \% of total energy }\end{array}$ & $6.96(5.78,8.52)$ & & & $6^{\mathrm{h}}$ & $70.11 \%$ \\
\hline \multicolumn{6}{|c|}{ 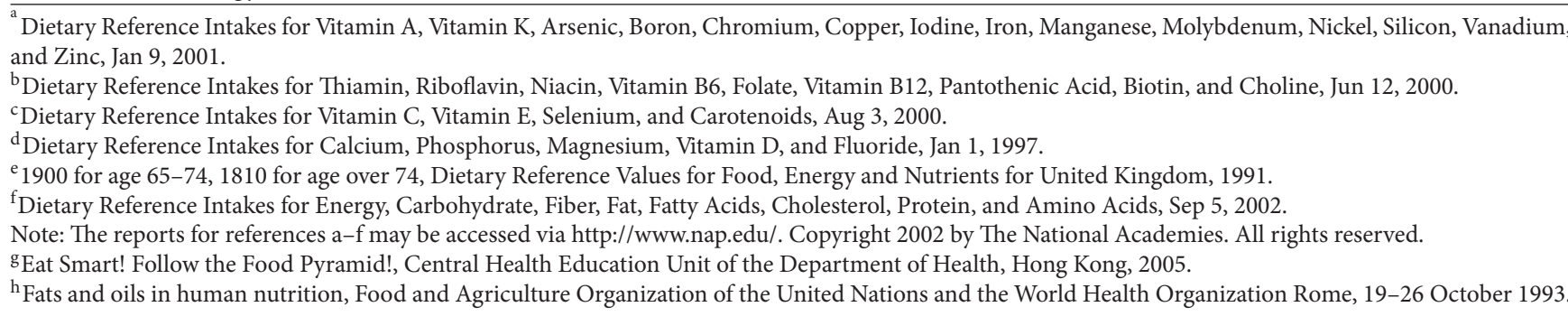 } \\
\hline
\end{tabular}


TABLE 4: Crude odds ratio and adjusted odds ratio of educational level in meeting nutrient recommendation.

\begin{tabular}{|c|c|c|c|c|}
\hline \multirow{2}{*}{ Educational level } & \multicolumn{2}{|c|}{ Female } & \multicolumn{2}{|c|}{ Male } \\
\hline & $\begin{array}{l}\text { Crude odds } \\
\text { ratio }(95 \% \mathrm{CI})\end{array}$ & $\begin{array}{l}\text { Adjusted by } \\
\text { logistic } \\
\text { regression } \\
(95 \% \mathrm{CI})\end{array}$ & $\begin{array}{l}\text { Crude odds } \\
\text { ratio }(95 \% \mathrm{CI})\end{array}$ & $\begin{array}{l}\text { Adjusted by } \\
\text { logistic } \\
\text { regression } \\
(95 \% \mathrm{CI})\end{array}$ \\
\hline \multicolumn{5}{|l|}{ Vitamin B1 } \\
\hline No education & 1 & 1 & 1 & 1 \\
\hline Primary or below & $1.23(0.95,1.61)$ & $1.20(0.89,1.60)$ & $1.80(0.97,3.35)$ & $1.67(0.90,3.13)$ \\
\hline $\begin{array}{l}\text { Secondary or } \\
\text { above }\end{array}$ & $2.02(1.47,2.77)$ & $1.93(1.36,2.75)$ & $3.12(1.68,5.81)$ & $2.84(1.51,5.32)$ \\
\hline \multicolumn{5}{|l|}{ Vitamin B2 } \\
\hline No education & 1 & 1 & 1 & 1 \\
\hline Primary or below & $1.64(1.29,2.07)$ & $1.51(1.17,1.95)$ & $1.35(0.78,2.36)$ & $1.24(0.71,2.18)$ \\
\hline $\begin{array}{l}\text { Secondary or } \\
\text { above }\end{array}$ & $2.91(2.19,3.87)$ & $2.65(1.94,3.62)$ & $2.31(1.33,4.02)$ & $2.08(1.18,3.65)$ \\
\hline \multicolumn{5}{|l|}{ Vitamin B3 } \\
\hline No education & 1 & 1 & 1 & 1 \\
\hline Primary or below & $1.31(1.04,1.64)$ & $1.19(0.93,1.52)$ & $1.15(0.76,1.75)$ & $1.12(0.73,1.74)$ \\
\hline $\begin{array}{l}\text { Secondary or } \\
\text { above }\end{array}$ & $2.40(1.82,3.16)$ & $2.13(1.57,2.89)$ & $1.92(1.26,2.94)$ & $1.74(1.12,2.71)$ \\
\hline \multicolumn{5}{|l|}{ Calcium } \\
\hline No education & 1 & 1 & 1 & 1 \\
\hline Primary or below & $2.08(1.03,4.23)$ & $1.70(0.82,3.51)$ & $1.85(0.44,7.77)$ & $1.61(0.38,6.81)$ \\
\hline $\begin{array}{l}\text { Secondary or } \\
\text { above }\end{array}$ & $3.30(1.52,7.19)$ & $2.87(1.26,6.50)$ & $\begin{array}{c}3.18(0.76 \\
13.31)\end{array}$ & $\begin{array}{c}2.93(0.70 \\
12.36)\end{array}$ \\
\hline \multicolumn{5}{|l|}{ Zinc } \\
\hline No education & 1 & 1 & 1 & 1 \\
\hline Primary or below & $1.41(1.15,1.72)$ & $1.21(0.97,1.50)$ & $1.14(0.73,1.78)$ & $1.09(0.69,1.73)$ \\
\hline $\begin{array}{l}\text { Secondary or } \\
\text { above }\end{array}$ & $2.50(1.92,3.25)$ & $1.98(1.49,2.64)$ & $1.60(1.02,2.51)$ & $1.45(0.91,2.32)$ \\
\hline \multicolumn{5}{|l|}{ Fruit } \\
\hline No education & 1 & 1 & 1 & 1 \\
\hline Primary or below & $1.17(0.94,1.46)$ & $1.13(0.89,1.44)$ & $1.81(1.11,2.94)$ & $1.65(1.00,2.70)$ \\
\hline $\begin{array}{l}\text { Secondary or } \\
\text { above }\end{array}$ & $1.91(1.45,2.51)$ & $1.84(1.36,2.49)$ & $2.20(1.34,3.60)$ & $1.97(1.19,3.26)$ \\
\hline \multicolumn{5}{|l|}{ Vegetable } \\
\hline No education & 1 & 1 & 1 & 1 \\
\hline Primary or below & $1.07(0.87,1.31)$ & $0.96(0.77,1.19)$ & $1.97(1.24,3.12)$ & $1.79(1.12,2.87)$ \\
\hline $\begin{array}{l}\text { Secondary or } \\
\text { above }\end{array}$ & $1.28(0.98,1.66)$ & $1.04(0.78,1.39)$ & $2.35(1.47,3.74)$ & $2.15(1.33,3.46)$ \\
\hline \multicolumn{5}{|l|}{ Meat } \\
\hline No education & 1 & 1 & 1 & 1 \\
\hline Primary or below & $1.16(0.93,1.45)$ & $1.04(0.82,1.33)$ & $1.11(0.74,1.66)$ & $1.05(0.69,1.60)$ \\
\hline $\begin{array}{l}\text { Secondary or } \\
\text { above }\end{array}$ & $1.55(1.17,2.04)$ & $1.26(0.93,1.72)$ & $1.46(0.97,2.21)$ & $1.31(0.85,2.02)$ \\
\hline \multicolumn{5}{|l|}{ Fibre } \\
\hline No education & 1 & 1 & 1 & 1 \\
\hline Primary or below & $1.72(1.10,2.69)$ & $1.89(1.14,3.11)$ & $1.14(0.54,2.43)$ & $1.10(0.52,2.35)$ \\
\hline $\begin{array}{l}\text { Secondary or } \\
\text { above }\end{array}$ & $2.48(1.48,4.15)$ & $2.46(1.37,4.42)$ & $1.58(0.74,3.35)$ & $1.46(0.68,3.13)$ \\
\hline
\end{tabular}


TABLE 5: Marital status as a risk factor in overintake of saturated and monounsaturated fatty acid of total energy and underintake of zinc and meat.

\begin{tabular}{|c|c|c|c|c|}
\hline \multirow{2}{*}{ Variable } & \multicolumn{2}{|c|}{ Female } & \multicolumn{2}{|c|}{ Male } \\
\hline & $\begin{array}{l}\text { Crude odds ratio } \\
\text { (95\% CI) }\end{array}$ & $\begin{array}{l}\text { Adjusted by logistic } \\
\text { regression }(95 \% \mathrm{CI})\end{array}$ & $\begin{array}{l}\text { Crude odds ratio } \\
(95 \% \mathrm{CI})\end{array}$ & $\begin{array}{l}\text { Adjusted by logistic } \\
\text { regression }(95 \% \mathrm{CI})\end{array}$ \\
\hline \multicolumn{5}{|c|}{$\begin{array}{l}\text { Meeting nutrient recommendation of } \\
\text { Zinc }\end{array}$} \\
\hline Single/divorced/widowed & $0.64(0.53,0.76)$ & $0.72(0.58,0.88)$ & $0.68(0.51,0.93)$ & $0.73(0.53,1.00)$ \\
\hline \multicolumn{5}{|c|}{$\begin{array}{l}\text { Meeting nutrient recommendation of } \\
\text { meat }\end{array}$} \\
\hline Single/divorced/widowed & $0.58(0.48,0.71)$ & $0.57(0.45,0.72)$ & $0.87(0.66,1.13)$ & $0.94(0.71,1.25)$ \\
\hline \multicolumn{5}{|c|}{$\begin{array}{l}\text { Over-intake of saturated fatty acid in total } \\
\text { energy }\end{array}$} \\
\hline Single/divorced/widowed & $0.89(0.50,1.58)$ & $0.84(0.43,1.65)$ & $1.86(1.12,3.09)$ & $1.91(1.11,3.31)$ \\
\hline
\end{tabular}

TABLE 6: Median/frequency (\%) among different age groups of the male participants.

\begin{tabular}{|c|c|c|c|c|}
\hline & \multicolumn{3}{|c|}{ Median/frequency (\%) } & \multirow[b]{2}{*}{$P$ value } \\
\hline & $65-69$ & $70-74$ & 75 or above & \\
\hline$N$ & 664 & 708 & 628 & \\
\hline Education level & & & & $<.0001$ \\
\hline No education & $27(4.07 \%)$ & $43(6.07 \%)$ & $33(5.25 \%)$ & \\
\hline Primary or below & $316(47.59 \%)$ & $401(56.64 \%)$ & $387(61.62 \%)$ & \\
\hline Secondary or above & $321(48.34 \%)$ & $264(37.29 \%)$ & $208(33.12 \%)$ & \\
\hline Single, divorced, or widowed & $47(7.08 \%)$ & $68(9.6 \%)$ & $125(19.9 \%)$ & $<.0001$ \\
\hline Living alone & $21(3.16 \%)$ & $26(3.67 \%)$ & $45(7.17 \%)$ & 0.0009 \\
\hline Weight & 63.80 & 62.80 & 61.00 & $<.0001$ \\
\hline Height & 163.95 & 162.95 & 162.30 & $<.0001$ \\
\hline BMI & 23.56 & 23.52 & 23.15 & 0.0009 \\
\hline \multicolumn{5}{|l|}{ Nutrient per day } \\
\hline Vitamin A $(\mu \mathrm{g})$ & 954.72 & 929.71 & 912.03 & 0.4011 \\
\hline Vitamin B1 (mg) & 0.88 & 0.89 & 0.86 & 0.1167 \\
\hline Vitamin B2 (mg) & 0.96 & 0.93 & 0.91 & 0.1474 \\
\hline Vitamin B3/niacin (mg) & 15.96 & 15.28 & 13.92 & $<.0001$ \\
\hline Vitamin C (mg) & 139.12 & 141.43 & 138.84 & 0.9536 \\
\hline Vitamin D ( $\mu \mathrm{g})$ & 0.23 & 0.19 & 0.17 & 0.0455 \\
\hline Calcium (mg) & 578.16 & 575.74 & 573.91 & 0.1867 \\
\hline Phosphorous (mg) & $1,079.64$ & $1,072.06$ & $1,018.04$ & 0.0032 \\
\hline Iron (mg) & 15.04 & 14.58 & 14.00 & 0.0028 \\
\hline Zinc (mg) & 9.86 & 9.63 & 9.28 & 0.0193 \\
\hline Iodine $(\mu \mathrm{g})$ & 0.34 & 0.30 & 0.25 & 0.0147 \\
\hline Copper $(\mu \mathrm{g})$ & 0.13 & 0.12 & 0.10 & $<.0001$ \\
\hline Total energy (Kcal) & $2,065.75$ & $2,050.85$ & $1,997.49$ & 0.0021 \\
\hline Protein $(\mathrm{g})$ & 84.12 & 81.66 & 78.17 & 0.0024 \\
\hline Protein $(\mathrm{g})$ /body weight $(\mathrm{kg})$ & 1.35 & 1.33 & 1.29 & 0.2816 \\
\hline Protein $\%$ of total energy & 16.50 & 16.07 & 15.89 & 0.0423 \\
\hline Carbohydrate (g) & 280.08 & 272.05 & 269.04 & 0.0534 \\
\hline Fruit (g) & 231.27 & 230.60 & 234.04 & 0.831 \\
\hline Vegetable (g) & 215.32 & 210.49 & 195.84 & 0.0741 \\
\hline Meat (g) & 151.83 & 147.12 & 139.55 & 0.0142 \\
\hline Fibre (g) & 8.68 & 8.72 & 8.27 & 0.3217 \\
\hline Cholesterol (mg) & 207.60 & 202.11 & 195.64 & 0.02 \\
\hline Fat $\%$ of total energy & 28.60 & 28.85 & 28.33 & 0.638 \\
\hline Saturated fatty acid $\%$ of total energy & 6.69 & 6.82 & 6.62 & 0.3391 \\
\hline Monounsaturated fatty acid $\%$ of total energy & 10.48 & 10.62 & 10.53 & 0.6339 \\
\hline Polyunsaturated fatty acid \% of total energy & 6.77 & 6.84 & 6.75 & 0.9904 \\
\hline
\end{tabular}


TABLE 7: Median/frequency (\%) among different age groups of the female participants.

\begin{tabular}{|c|c|c|c|c|}
\hline & \multicolumn{3}{|c|}{ Median/frequency (\%) } & \multirow{2}{*}{$P$ value } \\
\hline & $65-69$ & $70-74$ & 75 or above & \\
\hline$N$ & 669 & 665 & 666 & \\
\hline Education level & & & & $<.0001$ \\
\hline No education & $189(28.25 \%)$ & $259(38.95 \%)$ & $305(45.8 \%)$ & \\
\hline Primary or below & $334(49.93 \%)$ & $290(43.61 \%)$ & $279(41.89 \%)$ & \\
\hline Secondary or above & $146(21.82 \%)$ & $116(17.44 \%)$ & $82(12.31 \%)$ & \\
\hline Single, divorced, or widowed & $187(27.95 \%)$ & $273(41.05 \%)$ & $471(70.72 \%)$ & $<.0001$ \\
\hline Living alone & $66(9.87 \%)$ & $92(13.83 \%)$ & $183(27.48 \%)$ & $<.0001$ \\
\hline Housewife & $48(7.17 \%)$ & $60(9.02 \%)$ & $98(14.71 \%)$ & $<.0001$ \\
\hline Weight & 55.00 & 54.90 & 52.35 & $<.0001$ \\
\hline Height & 152.40 & 151.00 & 149.70 & $<.0001$ \\
\hline BMI & 23.61 & 24.15 & 23.34 & $<.0001$ \\
\hline \multicolumn{5}{|l|}{ Nutrient per day } \\
\hline Vitamin A $(\mu \mathrm{g})$ & 952.94 & 931.52 & 869.95 & 0.0049 \\
\hline Vitamin B1 (mg) & 0.81 & 0.78 & 0.73 & 0.0898 \\
\hline Vitamin B2 (mg) & 0.86 & 0.84 & 0.78 & 0.0013 \\
\hline Vitamin B3/niacin (mg) & 11.26 & 10.48 & 10.05 & $<.0001$ \\
\hline Vitamin C (mg) & 143.18 & 140.12 & 139.28 & 0.5009 \\
\hline Vitamin D $(\mu \mathrm{g})$ & 0.16 & 0.18 & 0.24 & 0.0113 \\
\hline Calcium (mg) & 545.03 & 543.82 & 495.06 & 0.0021 \\
\hline Phosphorous (mg) & 937.45 & 900.81 & 842.84 & 0.0003 \\
\hline Iron $(\mathrm{mg})$ & 12.16 & 11.62 & 11.09 & 0.0001 \\
\hline Zinc (mg) & 7.60 & 7.54 & 6.99 & 0.0003 \\
\hline Iodine $(\mu \mathrm{g})$ & 0.36 & 0.39 & 0.32 & 0.1767 \\
\hline Copper $(\mu \mathrm{g})$ & 0.09 & 0.07 & 0.06 & $<.0001$ \\
\hline Total energy (Kcal) & $1,550.90$ & $1,497.26$ & $1,463.53$ & 0.0002 \\
\hline Protein $(\mathrm{g})$ & 63.30 & 60.91 & 56.74 & $<.0001$ \\
\hline Protein (g)/body weight (kg) & 1.15 & 1.13 & 1.08 & 0.1801 \\
\hline Protein $\%$ of total energy & 16.18 & 16.01 & 15.64 & 0.0023 \\
\hline Carbohydrate (g) & 221.86 & 217.75 & 213.27 & 0.0471 \\
\hline Fruit (g) & 225.07 & 205.63 & 210.87 & 0.0897 \\
\hline Vegetable (g) & 220.46 & 211.91 & 192.34 & $<.0001$ \\
\hline Meat $(\mathrm{g})$ & 108.15 & 107.55 & 97.20 & 0.0004 \\
\hline Fibre $(\mathrm{g})$ & 8.32 & 8.02 & 7.56 & 0.0042 \\
\hline Cholesterol (mg) & 137.10 & 136.95 & 128.93 & 0.0058 \\
\hline Fat $\%$ of total energy & 27.09 & 27.16 & 26.10 & 0.1161 \\
\hline Saturated fatty acid $\%$ of total energy & 5.91 & 6.04 & 5.82 & 0.3437 \\
\hline Monounsaturated fatty acid \% of total energy & 10.03 & 9.84 & 9.76 & 0.0499 \\
\hline Polyunsaturated fatty acid \% of total energy & 6.90 & 6.91 & 7.04 & 0.6163 \\
\hline
\end{tabular}

individualized items of food and therefore does not capture some extreme diet range. Our study recruitment was not done by random sampling although we tried stratifying our subjects to cover a wider range of age and their BMIs are no different from the mean values of Hong Kong elderly. Our subjects are all ambulatory and therefore they cannot represent the elderly who live in institutions. Moreover, recruitment by self-referral usually will attract more health conscious and healthier subjects.

Our study has shown that there are nutritional dietary imbalances including under- and overconsumption of a wide range of nutrients in Hong Kong elderly. Diets have 
become westernized and rates of noncommunicable diseases increased. Our study has identified the health need of malnutrition problem in the elderly, and it also revealed that this problem existed across different socioeconomic classes. Education has played much influence on this health problem and therefore health promotion strategy should be adjusted according to the different educational levels. Health services should be targeted at promoting better diets and tackling barriers to healthier diet decisions.

\section{Conflict of Interests}

The authors declare that they have no conflict of interests.

\section{References}

[1] Keep Fit For Life: Meeting the Nutritional Needs of Older Persons, World Health Organization, 2002.

[2] C. La Vecchia, "Mediterranean diet and cancer," Public Health Nutrition, vol. 7, no. 7, pp. 965-968, 2004.

[3] J. M. Yuan, Q. S. Wang, R. K. Ross, B. E. Henderson, and M. C. Yu, "Diet and breast cancer in Shanghai and Tianjin, China," British Journal of Cancer, vol. 71, no. 6, pp. 1353-1358, 1995.

[4] F. B. Hu and W. C. Willett, "Optimal diets for prevention of coronary heart disease," Journal of the American Medical Association, vol. 288, no. 20, pp. 2569-2578, 2002.

[5] Y. Ruixing, W. Jinzhen, H. Yaoheng et al., "Associations of diet and lifestyle with hyperlipidemia for middle-aged and elderly persons among the Guangxi Bai Ku Yao and Han populations," Journal of the American Dietetic Association, vol. 108, no. 6, pp. 970-976, 2008

[6] J. Woo, S. C. Ho, and A. L. M. Yu, "Lifestyle factors and health outcomes in elderly Hong Kong Chinese aged 70 years and over," Gerontology, vol. 48, no. 4, pp. 234-240, 2002.

[7] G. D. Smith and E. Brunner, "Socio-economic differentials in health: the role of nutrition," Proceedings of the Nutrition Society, vol. 56, no. 1, pp. 75-90, 1997.

[8] I. Darnton-Hill and E. T. Coyne, "Feast and famine: socioeconomic disparities in global nutrition and health," Public Health Nutrition, vol. 1, no. 1, pp. 23-31, 1998.

[9] A. R. Suriah, T. J. Chong, and B. Y. Yeoh, "Nutritional situation of a Chinese community," Singapore Medical Journal, vol. 39, no. 8, pp. 348-352, 1998.

[10] J. Woo, C. K. Cheung, S. C. Ho, Y. T. Mak, and R. Swaminathan, "Protein nutritional status in elderly Chinese in Hong Kong," European Journal of Clinical Nutrition, vol. 42, no. 11, pp. 903-909, 1988.

[11] A. A. South Paul, McCance \&Widdowson's: The Composition of Foods, HMSO, London, UK, 4th edition, 1978.

[12] Y. Yang, G. Wang, and X. Pan, China Food Composition, University Medical Press, Peking, China, 2002.

[13] H. Cai, G. Yang, Y. B. Xiang et al., "Sources of variation in nutrient intakes among men in Shanghai, China," Public Health Nutrition, vol. 8, no. 8, pp. 1293-1299, 2005.

[14] J. Woo, S. S. F. Leung, S. C. Ho, T. H. Lam, and E. D. Janus, "A food frequency questionnaire for use in the chinese population in hong kong: description and examination of validity," Nutrition Research, vol. 17, no. 11-12, pp. 1633-1641, 1997.

[15] Hong Kong Cancer Registry, http://www3.ha.org.hk/cancereg/.

[16] http://www.iofbonehealth.org/.
[17] F. H. Fu, "The prevalence of cardiovascular disease risk factors of Hong Kong Chinese," Journal of Sports Medicine and Physical Fitness, vol. 41, no. 4, pp. 491-499, 2001.

[18] W. Lin and Y. W. Lee, "Nutrition knowledge, attitudes, and dietary restriction behavior of the Taiwanese elderly," Asia Pacific Journal of Clinical Nutrition, vol. 14, no. 3, pp. 221-229, 2005. 


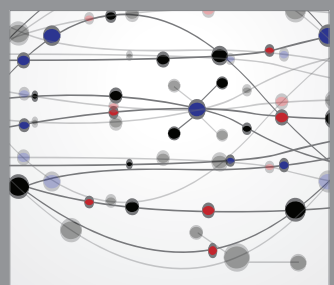

The Scientific World Journal
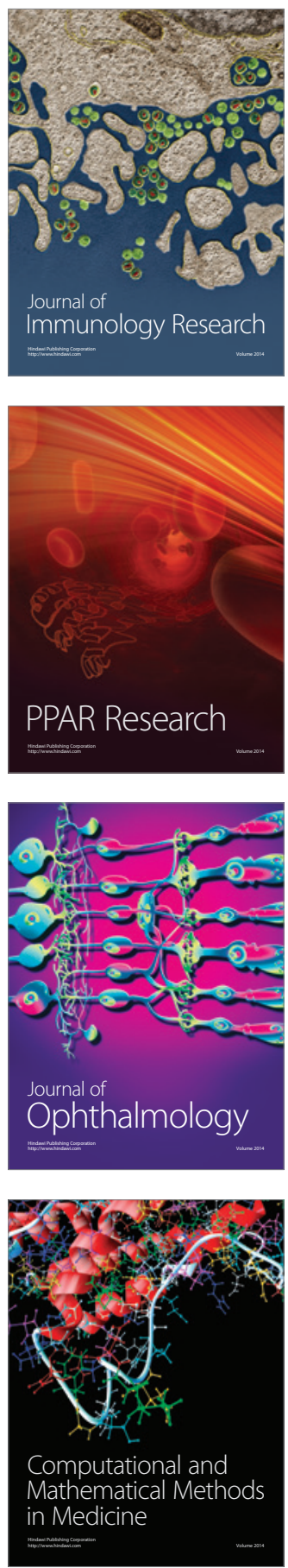

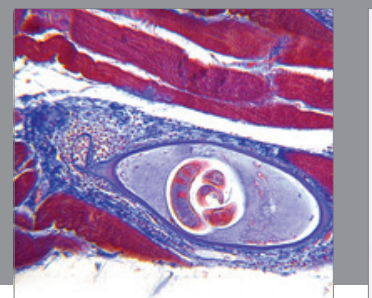

Gastroenterology

Research and Practice
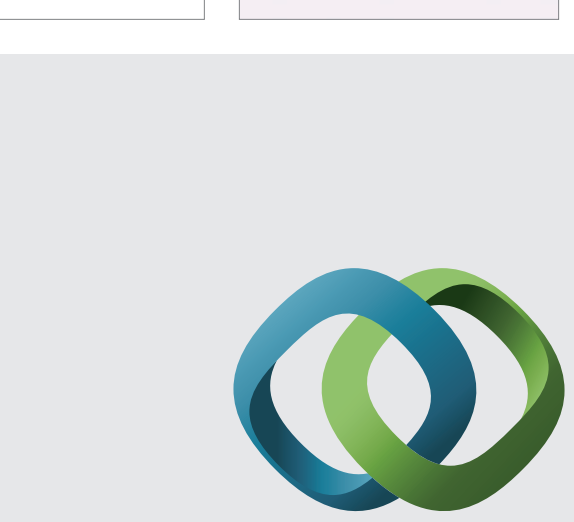

\section{Hindawi}

Submit your manuscripts at

http://www.hindawi.com
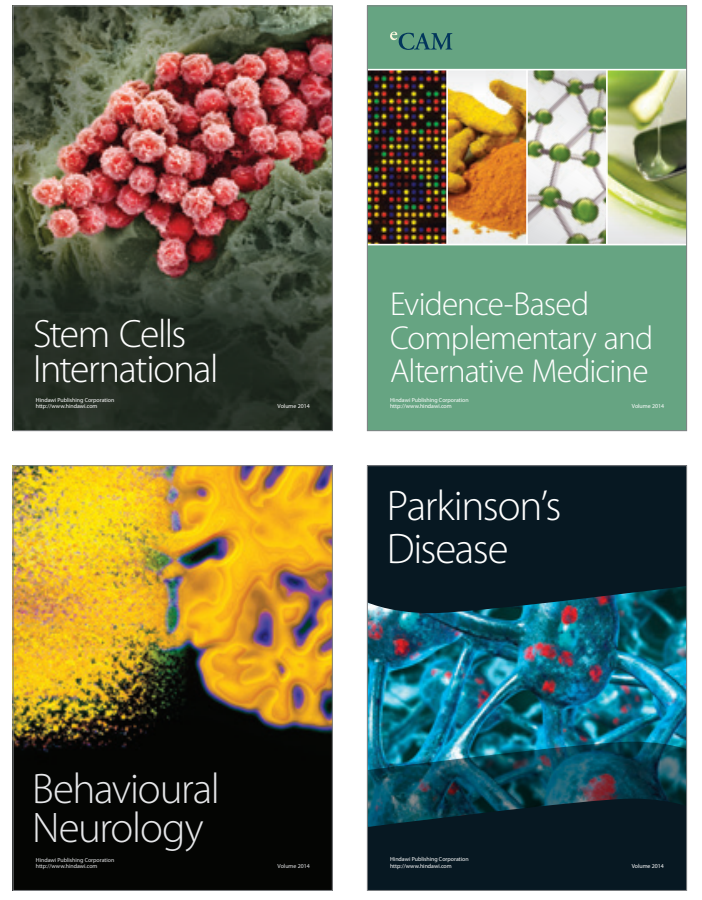
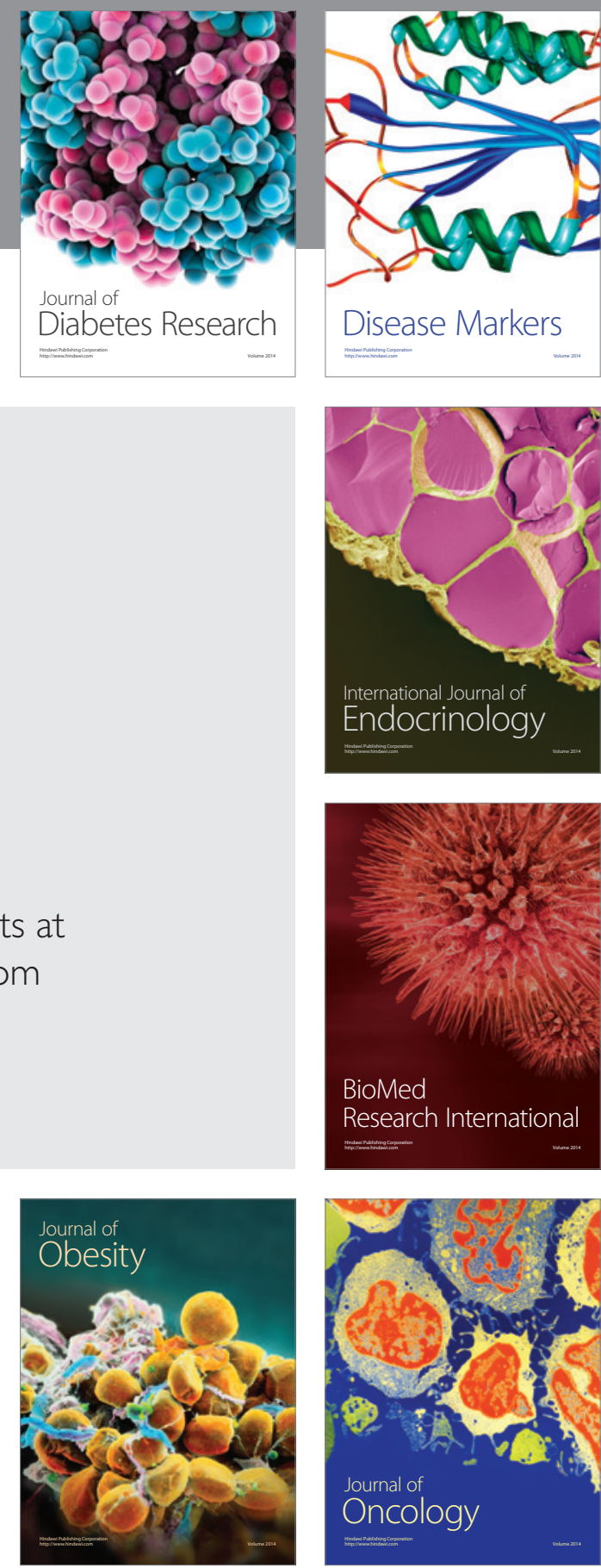

Disease Markers
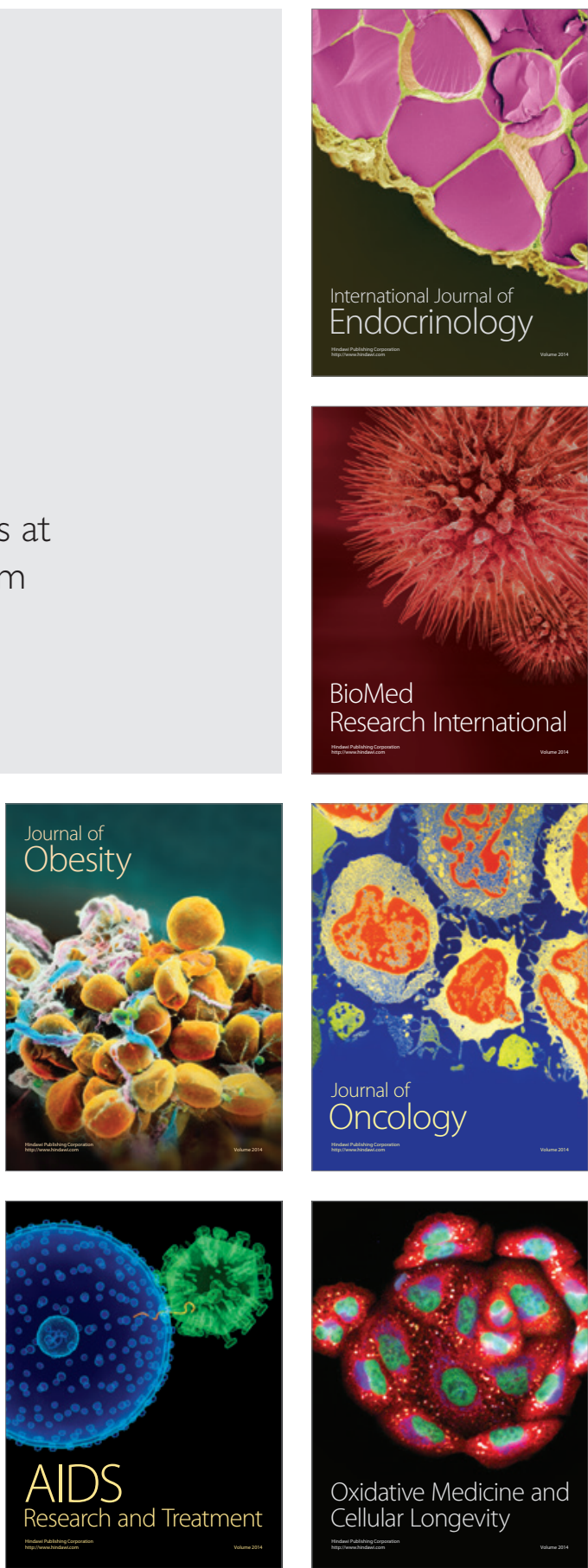